\title{
Dynamic behavior of superconducting flux qubit excited by a series of electromagnetic pulses
}

\author{
A.S. Kiyko, A.N. Omelyanchouk, and S.N. Shevchenko \\ B. Verkin Institute for Low Temperature Physics and Engineering of the National Academy of Sciences of Ukraine \\ 47 Lenin Ave., Kharkov 61103, Ukraine \\ E-mail: kiyko@ilt.kharkov.ua
}

Received July 17, 2007

\begin{abstract}
We study theoretically the behavior of the superconducting flux qubit subjected to a series of electromagnetic pulses. The possibility of controlling the system state via changing the parameters of the pulse is studied. We calculated the phase shift in a tank circuit weakly coupled to the qubit which can be measured by the impedance measurement technique. For the flux qubit we consider the possibility of estimating the relaxation rate from the impedance measurements by varying the delay time between the pulses.
\end{abstract}

PACS: 03.67.Mn Entanglement production, characterization, and manipulation;

74.25.Nf Response to electromagnetic fields;

85.25.Am Superconducting device characterization, design, and modeling;

85.25.Hv Superconducting logic elements and memory devices; microelectronic circuits.

Keywords: two-level systems, superconducting qubits.

\section{Introduction}

Quantum effects in mesoscopic superconducting circuits based on small Josephson junctions have attracted renewed attention. It has been demonstrated that Josephson devices at low temperature behave like quantum two-level systems. Therefore, ideas developed in atomic and molecular physics can be used for description of artificially fabricated circuits of macroscopic size. These concepts are stimulated further by the perspectives to realize quantum bits (qubits) for quantum information processing. Qubits are effective two-level quantum systems with externally controlled parameters. In the last decade a large number of proposals for building the qubits based on Josephson elements were proposed [1-4]. There are three basic types of Josephson-junction circuits that behave quantum-mechanically at low temperature. They are charge [1], phase [2], and flux [3] qubits. All of them can be fabricated with high precision with the help of modern lithography and can be the basis of the quantum computer. Promising for quantum computations is the $3 \mathrm{JJ}$ flux qubit that consists of the superconducting loop with three Josephson junctions [3]. This type of qubit is insensitive to charge noise, and it was shown that it has a high quality factor [5]. It was predicted that such systems should exhibit various quantum-mechanical effects in- cluding macroscopic quantum tunneling of the flux [6]. Indeed the predicted effects have been observed experimentally $[2,7,8]$. The quantum dynamics in single qubits was studied in $[3,4,9]$.

In our work we study the dynamics of the flux qubit subjected to a series of rectangular electromagnetic pulses. We present the model we use for calculations of the phase shift $\alpha$ in the resonant tank circuit based on the density matrix approach. Next we analyze the case which permits analytical solution and obtain small addition to the $\alpha$ in the ground state as function of the relaxation rate $\Gamma_{R}$. For arbitrary parameters we solve equations numerically and compare obtained result with analytical calculations.

\section{The model}

Our aim is to study the behavior of superconducting $3 \mathrm{JJ}$ flux qubit excited by a series of rectangular electromagnetic pulses. Flux qubit consists of a superconducting loop with three junctions: two identical and one with the parameters differing by factor $\beta$. For all calculations below we take $\beta$ equal to 0.8 .

The Hamiltonian of the flux qubit in the two-level approximation has the form $[10,11]$ : 


$$
\hat{H}=-\varepsilon \hat{\sigma}_{z}-\Delta \hat{\sigma}_{x},
$$

where the diagonal term $\varepsilon$ is the bias and the off-diagonal term $\Delta \propto \exp \left(-E_{J} / E_{C}\right)$ is the tunneling amplitude between the wells. Here $\hat{\sigma}_{x}, \hat{\sigma}_{z}$ are Pauli matrices in the basis $\{|\downarrow\rangle,|\uparrow\rangle\}$ of the current operator in the qubit: $\hat{I}=I_{0} \hat{\sigma}_{z}$, $I_{0}=I_{C} \lambda(\beta, g) / 2 \pi$, where $I_{C}$ is the critical current of the qubit, $g=E_{J} / E_{C}$, the explicit formula for the $\lambda(\beta, g)$ can be found in Ref. 12. The eigenstates of $\hat{\sigma}_{z}$ correspond to the clockwise $\left(\hat{\sigma}_{z}|\downarrow\rangle=-|\downarrow\rangle\right)$ and counterclockwise $\left(\hat{\sigma}_{z}|\uparrow\rangle=|\uparrow\rangle\right)$ currents in the qubit. The bias

$$
\varepsilon=I_{0} \Phi_{0}\left(f-\frac{1}{2}\right)
$$

is controlled by the dimensionless applied magnetic flux $f=\Phi_{x} / \Phi_{0}$ through the qubit; $\Phi_{0}=h / 2 e$ is the flux quantum.

The magnetic flux consists of two components:

$$
f=f_{D C}+\tilde{f}(t),
$$

which describe the adiabatically changing magnetic flux, $f_{D C}$, and the time-dependent component, $\widetilde{f}(t)$. We will study the possibility to control the system state via the series of the rectangular pulses with the amplitude $f_{A}$ and duration from $t_{1}^{(n)}=n(T+\tau)$ to $t_{2}^{(n)}=n(T+\tau)+T$ :

$$
\tilde{f}(t)=\sum f_{A}\left[\theta\left(t-t_{1}^{(n)}\right)-\theta\left(t-t_{2}^{(n)}\right)\right],
$$

where $\theta(t)$ stands for the theta-function, $T$ is the pulse duration, and $\tau$ is the delay between pulses (Fig. 1). The effect of the pulse is in changing the level occupation probabilities and to make them oscillating functions of time during the pulse. It should be noted that in the basis $\{|\downarrow\rangle,|\uparrow\rangle\}$ of the current operator, which are not eigenstates of the Hamiltonian, the probabilities oscillate both during and after the pulse.

We describe the system's evolution with the Bloch equation for the density matrix $\hat{\rho}(\hbar=1)$ :

$$
\frac{d \hat{\rho}}{d t}=-i[\hat{H}, \hat{\rho}]+\hat{\Gamma} \hat{\rho} .
$$

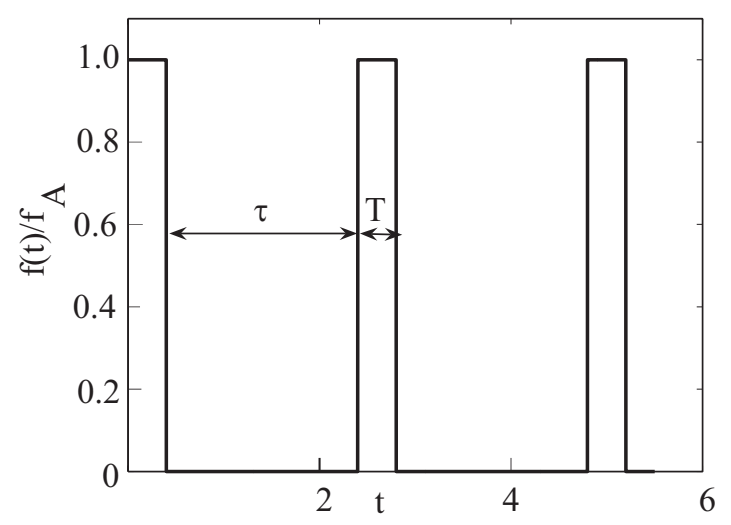

Fig. 1. Series of pulses
The impedance measurement technique $[13,14]$ consists in that the tank circuit probes the effective inductance of the system via measuring the phase shift $\alpha$ between the voltage and current in the tank circuit. The phase shift $\alpha$ is related to the Josephson inductance $\mathcal{L}$ of the qubit as follows:

$$
\begin{gathered}
\tan \alpha \approx k^{2} Q L \mathcal{L}^{-1}, \\
\mathcal{L}^{-1}=\frac{1}{\Phi_{0}} \frac{\partial\langle\hat{I}\rangle}{\partial f_{D C}}==\frac{I_{0}}{\Phi_{0}} \frac{\partial}{\partial f_{D C}} \operatorname{Sp}\left(\hat{\rho} \hat{\sigma}_{z}\right) .
\end{gathered}
$$

Here $M$ is the mutual inductance of the qubit with the tank circuit; $Q=R_{T} \sqrt{C_{T} / L_{T}}$, and $k=M / \sqrt{L L_{T}}$ are the quality factor and the coupling coefficient for the tank circuit, which consists of the inductor, $L_{T}$, capacitor, $C_{T}$, and resistor, $R_{T}$, connected in parallel (see in [15] for more details).

\section{Excitation of the flux qubit with the series of pulses}

In this Section we study the excitation of the flux qubit with the series of rectangular pulses. We start from the general 1-qubit Hamiltonian that has the form of Eq. (1) in the basis of states $\{|\downarrow\rangle,|\uparrow\rangle\}$, assuming $\widetilde{f}(t)=0$. For a flux qubit these states correspond to a definite direction of the current circulating in the ring. First the time-independent Hamiltonian is diagonalized in the basis of eigenstates $\{|-\rangle,|+\rangle\}$ with the rotation matrix $\hat{S}$ :

$$
\hat{S}=\left(\begin{array}{cc}
\cos (\eta / 2) & \sin (\eta / 2) \\
-\sin (\eta / 2) & \cos (\eta / 2)
\end{array}\right),
$$

with $\sin \eta=-\Delta \sqrt{\Delta^{2}+\varepsilon^{2}}, \cos \eta=\varepsilon / \sqrt{\Delta^{2}+\varepsilon^{2}}$.

For the calculation of the observable value, the phase shift $\alpha$ in the tank circuit, according to Eq. (6), we need the density matrix in the energy representation, where its diagonal components are equal to the probability of the system to be in the ground $|-\rangle$ or excited state $|+\rangle$.

Next we introduce the time-dependent terms into the time-independent Hamiltonian. Making use of the transformation $\hat{H}(t)=\hat{S}^{-1} \hat{H}(t) \hat{S}$, we get the Hamiltonian $\hat{H}(t)$ in the energy representation for the flux qubit [16]:

$$
\begin{gathered}
\hat{H}(t)=-\frac{\Delta E}{2} \hat{\tau}_{z}-2 I_{0} \Phi_{0} \tilde{f}(t)\left(\cos \eta \hat{\tau}_{z}+\sin \eta \hat{\tau}_{x}\right) / \Delta E, \\
\Delta E=2 \sqrt{\Delta^{2}+\varepsilon^{2}}
\end{gathered}
$$

The time evolution of the density matrix, which can be taken in the form $\hat{\rho}=\left(\hat{1}+X \hat{\tau}_{x}+Y \hat{\tau}_{y}+Z \hat{\tau}_{z}\right) / 2$, is described by the equation of motion (5). Initial condition for the density matrix in $\{|-\rangle,|+\rangle\}$ basis is $X(0)=Y(0)=0, Z(0)=1$, which corresponds to the ground state of the system. Solving the system of equations for $X(t), Y(t), Z(t)$ with 
phenomenologically introduced dephasing and relaxation rates $\Gamma_{\varphi}$ and $\Gamma_{R}$ :

$$
\begin{aligned}
& \frac{d X}{d t}=(\Delta E+h(t) \cos (\eta)) Y-\Gamma_{\varphi} X, \\
& \frac{d Y}{d t}=h(t) \sin (\eta) Z-(\Delta E+h(t) \cos (\eta)) X-\Gamma_{\varphi} Y, \\
& \frac{d Z}{d t}=-h(t) \sin (\eta) Y-\Gamma_{R}(Z-Z(0)),
\end{aligned}
$$

with $h(t)=2 I_{0} \Phi_{0} \tilde{f}(t)$, we obtain the probability of occupation of the upper level $|+\rangle$ (the excited state) $P_{+}(t)=$ $=\rho_{22}(t)=(1-Z(t)) / 2$. We calculate the density matrix in the flux basis making use of the transformation $\hat{\rho}_{\text {flux }}=\hat{S} \hat{\rho} \hat{S}^{-1}$ and obtain the probability of the current to be circulating in the clockwise direction $P_{L}(t)$, according to:

$$
P_{L}(t)=\frac{1}{2}(1-\sin (\eta) X(t)-\cos (\eta) Z(t)) .
$$

Averaging the $P_{L}(t)$ over $t$ we calculate phase shift $\alpha$ according to Eq. (6). For arbitrary values of the parameters of applied perturbation the system of equations (10) can be solved numerically. In the limiting case of one pulse (that is $\tau=\infty$ ) and without relaxation processes taking into account obtained results the solution can be found in Ref. 17 .

Before presenting the numerical results, consider the limiting case which permits the analytical solution:

$$
T<<T_{R, \varphi}=\Gamma_{R, \varphi}^{-1}<<\tau .
$$

In this case we can neglect the decay rates $\Gamma_{R}$ and $\Gamma_{\varphi}$ in (10) during the excitation time $T$ and assume that after the pulse during the delay time $\tau$ the system is returned to the ground state. Periodically repeating this process, we will have the input of relaxation processes in the time-averaged characteristics of the system.

With the assumptions (12) and for zero temperature the solution of the equations (10) can be found for the two time intervals: during the pulse $(0<t<T)$ and after the pulse $(T<t<T+\tau)$.

The solution for $0<t<T$ is the following:

$$
\begin{aligned}
& X_{1}(t)=\frac{2 A C}{A^{2}+C^{2}} \sin ^{2}\left(\frac{1}{2} \sqrt{A^{2}+C^{2}} t\right), \\
& Y_{1}(t)=-\frac{A}{\sqrt{A^{2}+C^{2}}} \sin \left(\sqrt{A^{2}+C^{2}} t\right), \\
& Z_{1}(t)=\frac{1}{A^{2}+C^{2}}\left(C^{2}+A^{2} \cos \left(\sqrt{A^{2}+C^{2}} t\right),\right.
\end{aligned}
$$

where $A=-h \sin \eta, C=-\Delta E-h \cos \eta, h=2 I_{0} \Phi_{0} f_{A}$; and for $T<t<T+\tau$ :

$$
\begin{aligned}
& X_{2}(t)=\exp \left[-\Gamma_{\varphi}(t-T)\right]\left[X_{1}(T) \cos (\Delta E(t-T))+\right. \\
& \left.+Y_{1}(T) \sin (\Delta E(t-T))\right], \\
& Z_{2}(t)=1-\exp \left[-\Gamma_{R}(t-T)\right]\left(1-Z_{1}(T)\right) .
\end{aligned}
$$

Let the duration of the pulse $T$ equals to $\pi / \sqrt{A^{2}+C^{2}}$, which corresponds to the one cycle of excitation during the time $T$.

Then we obtain:

$$
\begin{aligned}
& X_{2}(t)=\exp \left(-\Gamma_{\varphi}(t-T)\right) \frac{2 A C}{A^{2}+C^{2}} \cos (\Delta E(t-T)), \\
& Z_{2}(t)=1-\frac{2 A^{2}}{A^{2}+C^{2}} \exp \left(-\Gamma_{R}(t-T)\right) .
\end{aligned}
$$

Taking into account the inequalities (12), we obtain for the time-averaged values $\bar{Z}$ and $\bar{X}$ the following expressions:

$$
\begin{gathered}
\bar{X} \approx \frac{2 A C}{A^{2}+C^{2}} \frac{\Gamma_{\varphi}}{\left(\Gamma_{\varphi}^{2}+\Delta E^{2}\right) \tau}, \\
\bar{Z} \approx 1-\frac{2 A^{2}}{A^{2}+C^{2}} \frac{1}{\Gamma_{R} \tau} .
\end{gathered}
$$

Before substituting these values in the (11) and (16) we estimate the contribution of the $\bar{X}$ and $\bar{Z}$ into the phase shift $\alpha$ for the parameters we use for calculation. Our evaluation indicates that the contribution of the $\bar{X}$ is about three orders lower than that of the $\bar{Z}$, so we neglect the term containing $\bar{X}$ in Eq. (11). We calculate the small addition to the phase shift $\alpha$ due to the relaxation process $\alpha^{(1)}$. At the point $f=1 / 2$ we obtain after some algebra:

$$
\alpha^{(1)}=\frac{k^{2} Q L}{\Phi_{0}} \frac{2 f_{A}^{2}\left(I_{0} \Phi_{0}\right)^{3}}{\Delta\left(\left(I_{0} \Phi_{0} f_{A}\right)^{2}+\Delta^{2}\right)} \frac{1}{\tau \Gamma_{R}} .
$$

Hence from the measurement of the phase shift $\alpha$ at the point $f=1 / 2$ it is possible to estimate the relaxation rate according to Eq. (16). The behaviour of $\alpha^{(1)}$ at the point $f=1 / 2$ as the function of the product $\tau \Gamma_{R}$ is presented in Fig. 2. For the calculations we used the same parameters as for the numerical calculations below.

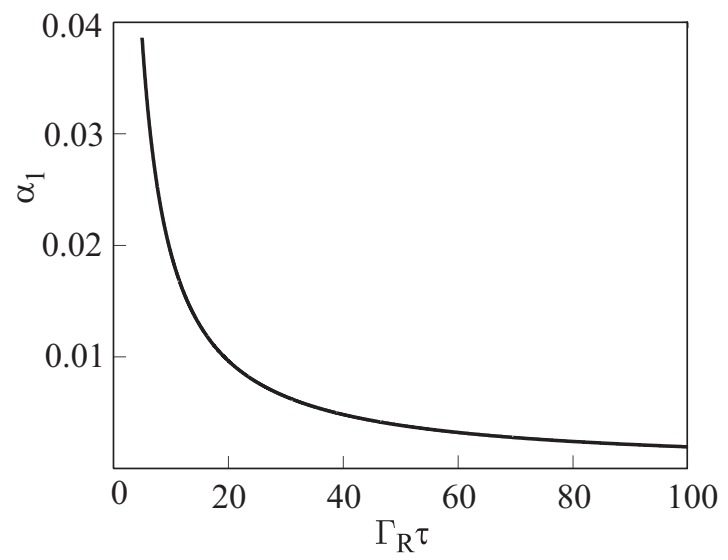

Fig. 2. The addition to the phase shift $\alpha_{1}$ for the flux qubit excited by the series of rectangular pulses due to the relaxation process in the point $f=0.5$ 

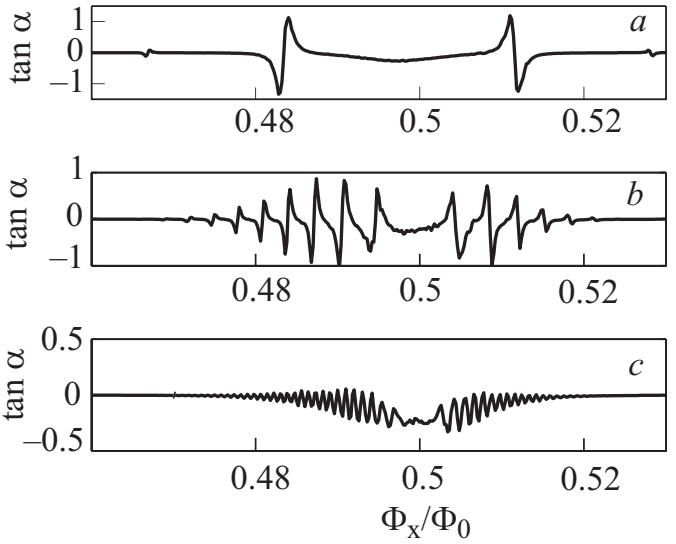

Fig. 3. The phase shift $\alpha$ for the flux qubit excited by the series of rectangular pulses with: $T=\tau=0.5(a), T=0.5, \tau=5(b)$, $T=0.5, \tau=20(c)$.

Now we study the excitation of the flux qubit by the series of rectangular pulses numerically. Namely we calculate the phase shift in the tank circuit by making use of the solution of the Eqs. (6) and (10). Fig. 3 is plotted for the following set of parameters for the qubit: $I_{0} \Phi_{0}==200 \mathrm{GHz}, \Delta=1.4 \mathrm{GHz}$, $k^{2} Q\left(L I_{0}\right) / \Phi_{0}=2 \cdot 10^{-3}$; the excitation $\tilde{f}(t)$ was considered to be the series of the pulses with $\tau=T<<\Gamma^{-1}, \tau=0.5 \Gamma^{-1}$, $\tau=2 \Gamma^{-1}$ (from upper to down) and the decay rates $\Gamma_{R}=\Gamma_{\varphi}=\Gamma=0.1 \mathrm{GHz}$. We observe that at $\tau \gtrsim \Gamma^{-1}$ the resonances disappear with increasing delay time $\tau$. This can be used in practice for relatively simple estimation of the decay rates by changing the delay time between the pulses.

Next we compare the theoretically (dashed) and numerically (marked by points) calculated curves for the phase shift $\alpha$ calculated under the assumption (12) from the (14) and (15). For the numerical calculations we use the following parameters of the pulse: $T=0.5, \tau=100, f_{A}=0.005$ and of the decay rates $\Gamma_{R}=\Gamma_{\varphi}=\Gamma=0.1 \mathrm{GHz}$. Such values of $\tau, T$ and $\Gamma_{R, \varphi}$ correspond to the limiting case which we considered previously (12), and one can see very good agreement in Fig. 4.

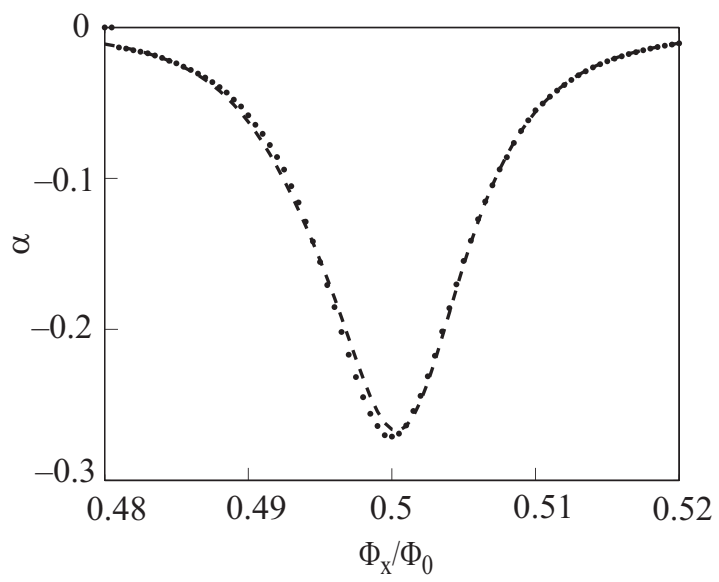

Fig. 4. Comparison of the theoretical (dashed) and numerical (marked by points) curves for the ecxitation with the series of the pulse with parameters: $T=0.5, \tau=100$.

\section{Conclusion}

Dynamics of a flux qubit subjected to a series of rectangular electromagnetic pulses. We investigated the changes of the tank circuit phase shift $\alpha$ for the single qubit that appears due to excitation by the pulses. It was demonstrated that the response of the tank circuit essentially depends on the relation between the decay rates $\Gamma_{R, \varphi}$ and the delay time $\tau$, which may be used for the estimation of $\Gamma_{R}$ by measuring the phase shift $\alpha$ as a function of the delay time $\tau$.

We would like to thank M. Grajcar and E. Il'ichev for helpful discussions. A.N.O. acknowledges the partial financial support of DFG grant. S.N.S. acknowledges the financial support of INTAS under the Fellowship Grant for Young Scientists (No. 05-109-4479).

1. Y. Nakamura, Y.A. Pashkin, and J.S. Tsai, Nature (London) 398, 786 (1999).

2. J.R. Friedman, V. Patel, W. Chen, S.K. Tolpygo, and J.E. Lukens, Nature (London) 406, 43 (2000).

3. J.E. Mooij, T.P. Orlando, L. Levitov, Lin Tian, Caspar H. van der Wal, and Seth Lloyd, Science 285, 1036 (1999).

4. D. Vion, A. Aassime, A. Cottet, P. Joyez, H. Pothier, C. Urbina, D. Esteve, and M.H. Devoret, Science 296, 886 (2002).

5. C.H. van der Wal, F.K. Wilhelm, C.J.P.M. Harmans, and J.E. Mooij, Eur. Phys. J. B31, 111 (2003); L. Tian, S. Lloyd, and T.P. Orlando, Phys. Rev. B65, 144516 (2002).

6. A.O. Caldeira and A.J. Leggett, Ann. Phys. (N.Y.) 149, 374 (1983).

7. J. Clarke, A.N. Cleland, M.H. Devoret, D. Estive, and J.M. Martinis, Science 239, 992 (1988).

8. C.H. van der Wal, A.C.J. ter Haar, F.K. Wilhelm, R.N. Schouten, C.J.P.M. Harmans, T.P. Orlando, S. Lloyd, and J.E. Mooij, Science 290, 773 (2000).

9. I. Chiorescu, Y. Nakamura, C.J.P.M. Harmans, and J.E. Mooij, Science 299, 1869 (2003).

10. Y. Makhlin, G. Schön, A.Shnirman, Rev. Mod. Phys. 73, 357 (2001).

11. M. Grajcar A. Izmalkov, E. Il'ichev, Th. Wagner, N. Oukhanski, U. Hübner, T. May, I. Zhilyaev, H.E. Hoenig, Ya.S. Greenberg, V.I. Shnyrkov, D. Born, W. Krech, H.-G. Meyer, Alec Maassen van den Brink, and M.H.S. Amin, Phys. Rev. B69, 060501 (2004).

12. Y.S. Greenberg, A. Izmalkov, M. Grajcar, E. Il'ichev, W. Krech, H.G. Meyer, M.H.S. Amin, and A. M. van den Brink, Phys. Rev. B66, 214525 (2002).

13. R. Rifkin and B.S. Deaver, Jr., Phys. Rev. B13, 3894 (1976).

14. E. Il'ichev, V. Zakosarenko, L. Fritsch, R. Stolz, H.E. Hoenig, H.-G. Meyer, M.Götz, A.B. Zorin, V.V. Khanin, A.B. Pavolotsky, and J. Niemeyer, Rev. Sci. Instr. 72, 1882 (2001).

15. S.N. Shevchenko, to be published.

16. S.N. Shevchenko, A.S.Kiyko, A.N. Omelyanchouk, W. Krech, Fiz. Nizk. Temp. 31, 752 (2005) [Low Temp. Phys. 31, 564 (2005)].

17. A.S. Kiyko, A.N. Omelyanchouk, and S.N. Shevchenko, in: Proceedings of $M S+S$ Conference, Kanagawa, Japan (2006). 